\title{
Comparison of ultrasound and LIB generated cavitation bubble
}

\author{
Darina Jasikova ${ }^{*}$, Petr Schovanec, Michal Kotek, Milos Muller, Vaclav Kopecky \\ Department of Physical Measurement, Technical University of Liberec, The Institute for Nanomaterials, Advanced Technology and \\ Innovation, Studentska 1402/2 Liberec 1461 17, Czech Republic
}

\begin{abstract}
There has been tried many methods how to generate single cavitation bubble. The purpose of these experiments is to observe the surface impact of collapsing cavitating bubbles. The most of method are based on spark to induced superheat limit of liquid. Here we compare the one of these methods - laser induced breakdown (LIB) with more correct mechanical way using ultrasound as generator. There is described the setup for ultrasound cavitation effecting the sessile bubble as well as random generated and moving bubble. We visualize the cavitation bubble using high speed camera of speed $180 \mathrm{kHz}$. There are observed interface instabilities as the ultrasound response.
\end{abstract}

\section{Introduction}

The cavitation is in the scope of not only industrial application, but also biomedical such as medicine, biology, pharmacy, or tissue engineering. The very popular became ultrasonic cavitation in these days. It has a potential in drug delivering systems, cell walls disintegrations, or viruses and bacteria immobilization.

It may seem that the subject dealing with cavitation has reoriented, as applications are concerned. It is not, in engineering applications with more research it has shifted toward solving direct interactions of cavitating bubbles. This take shape in creating novel resistant materials, testing and development of new, more resistant structures or layers which better withstand the action of cavitating bubbles and prolong the service life of products. The research moved to the mathematical modelling and physical analysing.

Anyway, we dealing with great technique, there still exists a lack of information in experimental part of cavitating bubble investigation. There are many ways how to wake cavitating bubble, or the cloud of bubbles. Only few of them follow the postulate of cavitation bubble theory.

The most common method for bubble generation is spark in liquid, or a heated top of the wire. Once the bubble is stable, and of certain volume, it is either over heated, or expose to force impact. Convenient method for generating single cavitation bubble that can be very precisely geometrically placed in the volume of the liquid, and close to the sample, is Laser Induced Breakdown (LIB). [1-5]

To be consistent, cavitation bubble is wake through local decrease of pressure that is ordinary caused by local velocity increase of the liquid. The local velocity increase can be induced by acoustic wave or other mechanical acting. The generation of the single cavitation bubble with acoustic wave is challenging.
There has to implemented conditions such as optimal basic wave frequency, the suitable amplitude, the acoustic wave dumping, and the dominating frequency taking in account the lifespan of cavitation bubble. We face many technical problems during implementation: the efficient power source working on variable set ultrasonic frequencies, the synchronizing elements, and the material resistivity. [6]

Cavitation can be defined as a collection of effects connected to the origin, activities, and collapse of macroscopic bubbles in liquid. Cavitation bubbles are usually not separated in real applications. The bubbles create structures, which acts collectively, however essential elements of these structures are individual bubbles.

The understanding of its dynamics and impact to the surroundings represents the key in the understanding of the cavitation phenomena in its complexity. Here we compare two methods of the impacting cavitation bubble. The first method is ultrasonic cavitation (SONO), the second LIB. The purpose of this study is to insight into the behaviour of bubbles.

\section{Experimental}

The bubbles can be generated by several physically different mechanisms. The most obvious in nature is the hydrodynamics cavitation, where bubbles are produced due to local pressure decrease caused by the flow acceleration in vicinity of obstacles. Acoustic cavitation is produced by imposing an intensive acoustic field into the bulk of liquid [1].

The acoustic field cause the local tension of the liquid and its rupture. Energy deposition represents another possibility for the bubble generation. Here, we used two ways for cavitation bubble generating in liquid. The spark based method LIB was compared to ultrasonic

Corresponding author: darina.jasikova@tul.cz 
wave impact to cavitating bubble. The working liquid was distillate water of $8 \mathrm{mg}$ dissolved $\mathrm{O}_{2} / 1$ at $20^{\circ} \mathrm{C}$. Both experiments run in optical glass cuvette.

\subsection{Laser Induced Breakdown}

Optical breakdown in liquid is usually produced by focusing of the laser light trough suitably designed optics. Laser induced breakdown in aqueous media and its collateral effects are described in detail by Kennedy in [2]. The energy distribution during the growth and collapse of laser induced bubble was described e.g. by Vogel in [7] and [8]. Authors investigated the influence of laser pulse duration and input laser energy on the bubble dynamics and shock waves emission.

For the single bubble generation we used here the setup for LIB. The 10ns width laser pulse was generated using Q-switched Nd:YAG NewGemini pulse laser. This laser worked with one cavity for single shot generation on the wavelength $532 \mathrm{~nm}$. The Q-switch signal synchronized the high speed camera running in triggering mode.

LIB was set as an optical direct way. The outlet diameter of laser beam was $5 \mathrm{~mm}$ with Gaussian characteristics of the intensity. This setup was followed with concave lens $f_{s}=200 \mathrm{~mm}$, and convex lens $\mathrm{f}_{\mathrm{s}}=25$ $\mathrm{mm}$ of 1 inch diameter. The focused laser beam created the laser point - probe (diameter $<0.1 \mathrm{~mm}$ ). Due the losses in the optical path on each of optical elements, comparable to this, we had to increase the energy level that enters the whole system. The set output energy of the laser is taken in account in the relation to the bubble diameter. The energies that are required for the bubble generation are seen in Fig. 1.

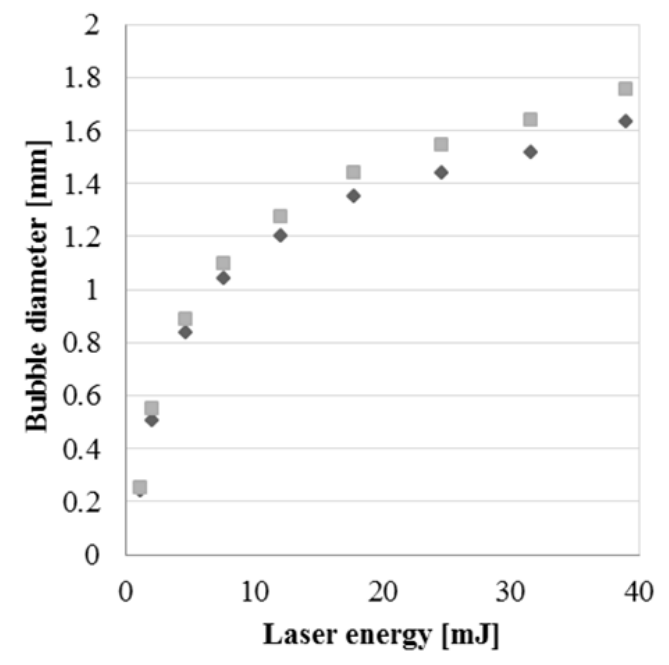

Fig. 1. The graph of maximal bubble diameter on laser energy.

For the relation between input laser energy and bubble size that was generated with our optical setup, we create dataset of size - measurement. The energy of laser beam was set and measured with Ophir pyroelectric energy sensor. The dataset of at least 20 cavitation processes for statistic evaluation was captured. This maximum bubble size was detected in each image and it was related to the energy of the laser beam. The bubble size was measured in vertical and horizontal axis and final value represents average value. The increase of laser energy causes the increase the horizontal value of cavitation bubble size. This effect corresponds with temporal and spatial plasma evolution at the very beginning of the process. The relation between the energy of laser beam and cavitation bubble size is asymptotic. This means that further increase of laser energy does not lead to significant increase of the bubble size.

According to Kennedy [9], plasma temperature shows asymptotic dependence on laser pulse energy as well. With higher laser energy we recognized negative influence of impurities and presence of segmentations on the bubble surface.

\subsection{SONO setup}

Ultrasound is an acoustic wave of frequency higher than $20 \mathrm{kHz}$. Its source can be mechanical, magnetostriction and piezoelectric generators.

Ultrasound passes through the material environment through the vibration spread over particles. Velocity of propagation is not frequency dependent, so the ultrasound propagates at same speed as sound. There are large differences in absorption in various environments. Ultrasound is strongly absorbed by gas, $100 \mathrm{kHz}$ frequency reach $2.2 \mathrm{~m}$ in the air, and in water $4 \mathrm{~km}$, whilst with lower wavelength absorption grows rapidly, so e.g. $1 \mathrm{MHz}$ ultrasound reach only $22 \mathrm{~mm}$ in the air and $40 \mathrm{~m}$ in water. [10]

We used the ultrasonic setup for observing and visualization of the processes mentioned above. The cuvette was fulfilled with water, and there was place sonotrode above the steel sample. The ultrasound was generated using ultrasonic Processor UIP1000hd (1000W) working on $20 \mathrm{kHz}$ frequency. The whole setup consisted of transducer and generator, and titanium horn as the sonotrode.

Cloud of bubbles was generated with te syringe pumping air into the liquid. Once the bubbles were spread close to the surface the ultrasonic generator was switched on.

\subsection{Visualization setup}

We used here shadowgraphy setup for the bubble visualization. This setup consists of continuous LED matrix daylight lamp Veritas, MiniConstellation 120 $5000 \mathrm{~K}$ of illuminance $92 \mathrm{klux}$ in $0.5 \mathrm{~m}$, set with optical diffuse filter. Opposite to the light source was placed high speed CMOS camera SpeedSense. This camera is working on frequency $180 \mathrm{kHz}$ with resolution of $(128 \mathrm{x}$ 128) px or lower frequency with higher resolution up to $(1280 \times 800) \mathrm{px}$, and the dynamic range $12 \mathrm{bit}$. The camera exposure time was $1 \mu \mathrm{s}$. The sub-pixel resolution was $20 \mu \mathrm{m}$. The camera was mounted with optical lens system INFINIPROBETM TS-160 universal macro/micro imaging system that enables $4 \mathrm{x}$, and 16x magnification. The camera was mounted with edge pass and long pass 
filter cut-on wavelength $550 \mathrm{~nm}$ low pass optical filter to reduce the backward laser flashes to the camera and also to eliminate the flash generated while plasmatic breakdown.

We also used a light reflecting setup for visualization of the outer bubble structures. We placed the light under $45^{\circ}$ from the camera direction.

\section{Results}

The ultrasonic cavitation means active manifestations of oscillating gas bubbles in the liquid induced by ultrasound waves. Ultrasound causes periodic changes in the instantaneous pressure in the liquid, which are superimposed on the pressure. The small gas bubbles presented in the liquid begin to radically nonlinearly oscillate at certain pressured amplitude. The cavitation can be divided between collapse (transient) and resonant (stable). However, the resonating cavitation bubble can go to chaotic behaviour and collapse after a few cycles. Therefore the system is marked as cavitating or not. There have been discovered two main sources of physical damage. The first source is pressure pulse emitted into the liquid near the collapsing bubbles.

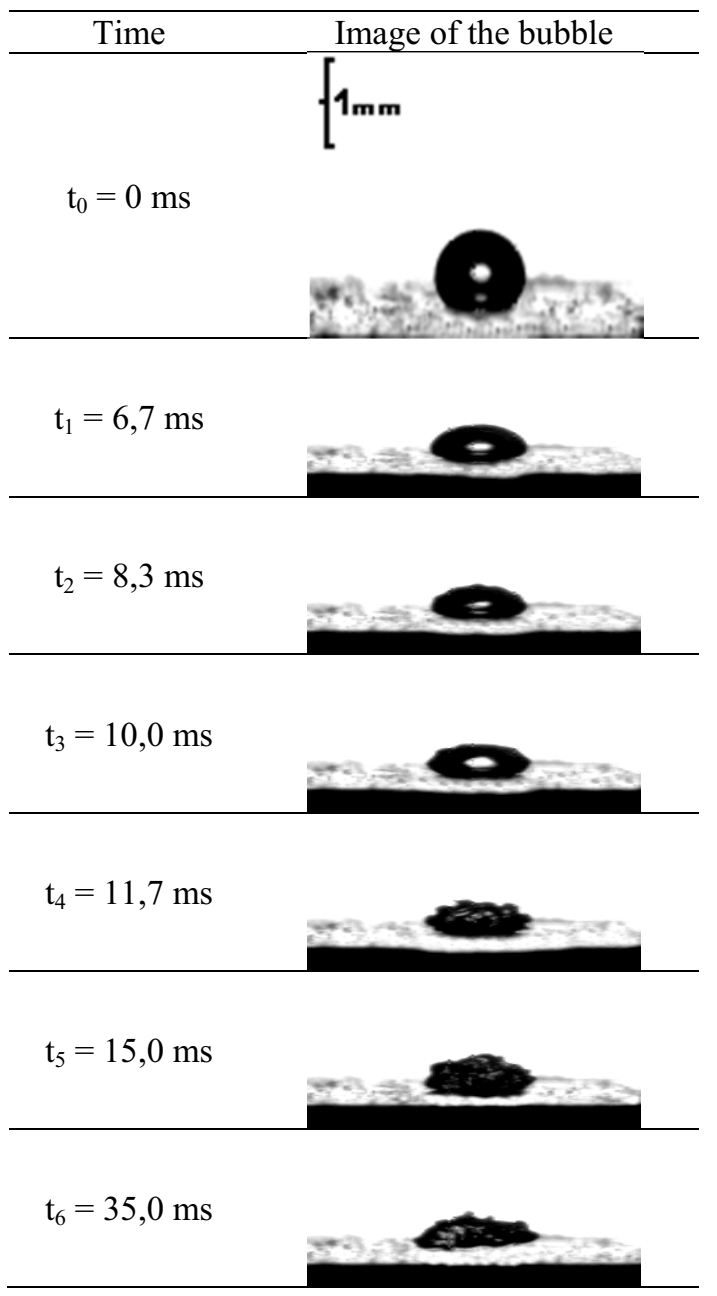

Fig. 2. The effect of the ultrasound on the sessile bubble using shadowgraph method.
The spherical shock wave may have amplitude of up to $1 \mathrm{GPa}$. When the bubble collapses isolated, the shock wave is quickly suppressed. This bubble is damaging only touching surface structure in the immediate vicinity. $[11,12]$ On the other hand, in a concentrated burst of bubbles the collapse of one bubble may induce the collapse of other bubbles around. This "cavitation cloud" can cause mechanical damage at greater distances from the "epicentre". [13]

The ultrasound effecting on the bubble only in the peak of amplitude that is of $20 \mathrm{kHz}$, so one time period is $50 \mathrm{~ms}$. Till the inner bubble pressure increase the certain value and collapse of bubble happens it takes a couple of periods.

The sessile bubble is directly acting at the surface. Finally, this leads to the bubble disintegration and breakup. For the better visualization of the bubble interface we used the reflective illumination method, the pictures taken with high speed camera are seen in Fig. 3. In this case, the bubble was directly generated with ultrasonic waves and moved towards the surface. The taken pictures are result of very stochastic process.

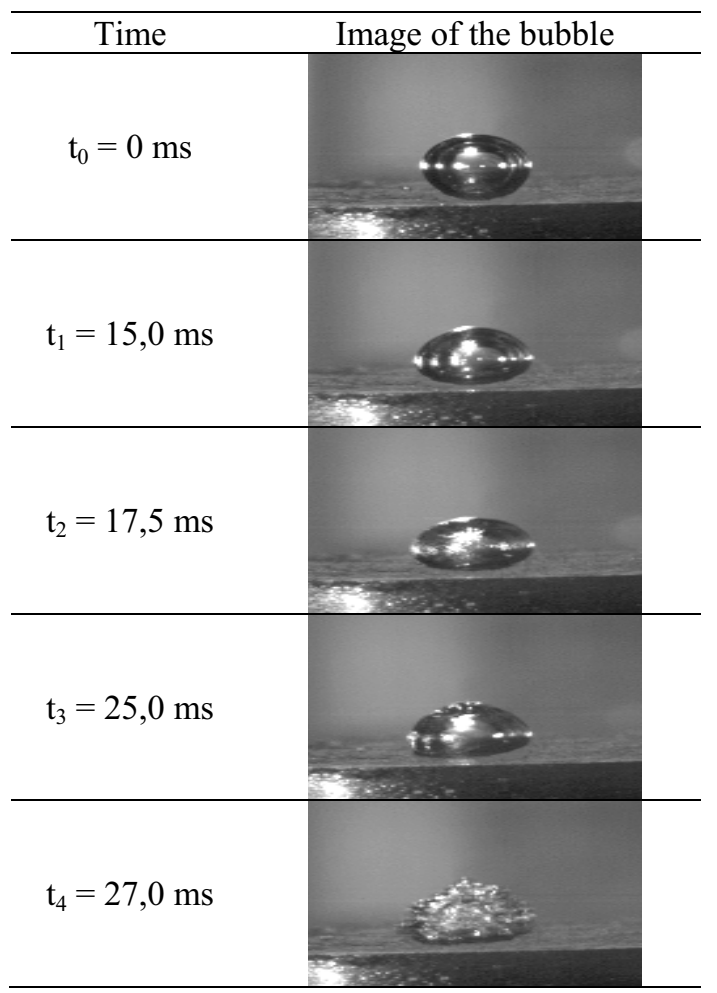

Fig. 3. The effect of the ultrasound on the sessile bubble using light reflecting method.

The structures of the gas - liquid interface are se as the orange-peel disturbance that is said to be caused by evaporations. Frost, et.al used this term firstly. [6] The structure is seen in Fig. 2 at time $t_{4}=11.7 \mathrm{~ms}$ from the Sono switch on. The instabilities are likewise RayleighTaylors. We managed to capture the very beginning of the instability spreading from the top of the bubble. This more follows the behaviour of the ultrasound as it spreads over the liquid and refracts and reflects from the cuvette walls and the sonotrode itself. The force of waves is summed in small locals and it's projected on 
the bubble interface. The lifespan of the bubble toward the disintegration is strongly dependent on source frequency. There is seen the bubble response on the first half of the ultrasound wave. The main influence has positive part of the amplitude on emergence of failures.

The Sono bubble of comparable size has lifespan in one order more than LIB generated one. This can be change using $2.3 \mathrm{MHz}$ ultrasound source, but of sufficient power.

Generate single cavitation bubble with LIB is limited by initial plasma probe. The geometry of the plasma predetermines the number of bubbles. If there is higher input of light energy the probe elongate and the energy diversify and there will be created more than one bubbles that finally conjunct into big one and collapse.

The LIB bubble lifespan is up to $120 \mu \mathrm{s}$. During the LIB the pressure increase is induced by the local temperature. This effect last $50 \mu \mathrm{s}$ in average. The bubble capturing starts at the plasma point that is set as the zero time.

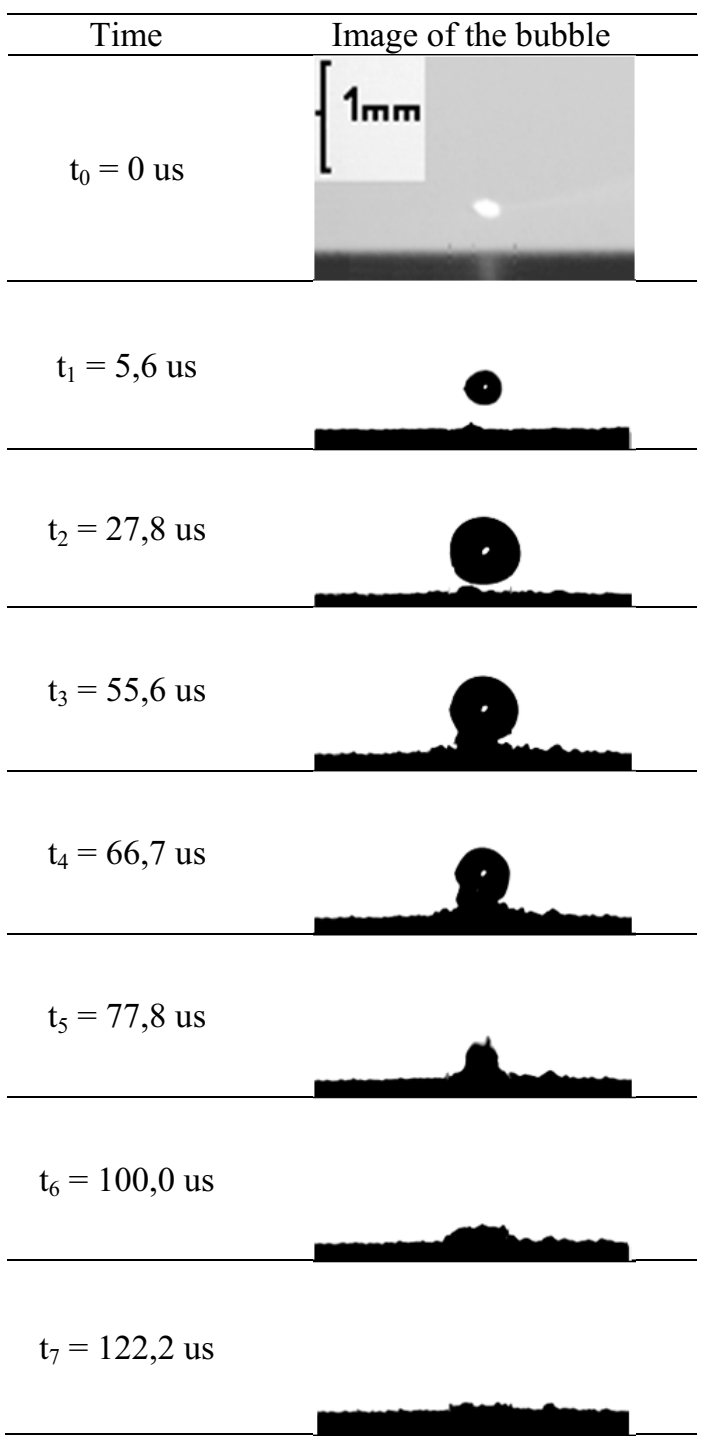

Fig. 4. LIB generated bubble and its implosion towards the surface.

The LIB bubble surface oscillations are developing faster than we were able to recognize. The maximum limit of the high speed camera was $180 \mathrm{kHz}$ to keep sufficient imager resolution. The assumption of surface oscillations is supported by the observation that the bubble is exploded unsymmetrically. This theme should be of further examination at the edge of technical limits.

\section{Conclusions}

There has been done a series of experiments as the ultrasound cavitation is random process. The bubble response to the mechanical action is dependent of the upper half of amplitude and frequency. The lifespan of the cavitation bubble is also dependent on the working frequency. As the interface instabilities are seen right as the reaction on the force impact we suppose that the orange-peel disturbance is caused by ultrasound wave refractions and reflections.

The comparison with LIB bubbles is varying in range of lifespan. Also the range of bubble size is limited to the plasma probe geometry unlike the ultrasound is stochastic effect. The bubbles are created in the range of sizes and mostly occur as the cloud of bubbles. The comparable bubbles can be generated only with increased ultrasound frequency of with the pulse, amplitude modulation, and focused sonotrode.

Here, we managed to visualize single cavitation bubble that is affected by the ultrasonic waves. This first step is crucial for further investigation of the process itself in term of measuring cavitation impact force on the sample of material.

The results of this project LO1201 were obtained through the financial support of the Ministry of Education, Youth and Sports in the framework of the targeted support of the "National Programme for Sustainability I". Authors also acknowledge the institutional support of the Faculty of Mechatronics, Informatics and Interdisciplinary Studies of the Technical University of Liberec.

\section{References}

1. Ch. E. Brennen, Cavitation and bubble dynamics, (Cambridge University Press, 2015)

2. P. K. Kennedy et.al, Prog.QuantElectr. 21, 3, (1997)

3. E. A. Brujan, et.al, J.Fluid Mech. 433, (2001)

4. W. Lauterborn, et.al, Archives of Acoustics 33, (2008)

5. A. Shima, Y. Tomita, Ingenieur-Archiv 51, (1981)

6. D. Frost, B. Sturtevant, ASME 108, (1986)

7. A. Vogel, S. Busch, U. Parlitz, J. Acoust. Soc. Am. 100, (1996)

8. A. Vogel, et. al, Applied Physics B: Lasers and Optics 68, (1999)

9. P. K. Kennedy et al., Prog. Quant. Electr. 21, 3, (1997)

10. I. Hrazdira, V. Mornstein, Medical biophysics and technology (Neptun, 2001)

11. R. Hickling, M. S. Plesset, Phys. Fluids, 7 (1964)

12. R. D. Ivany, Tech. Rep, 15, University of Michigan, Dept. Of nuclear engineering, 1965

13. B. Vias, C. M. Preece, J. Appl. Phys., 47 (1976) 